\title{
Human heat shock protein 27 exacerbates ischemia reperfusion injury in rats by reducing the number of $T$ regulatory cells
}

\author{
SUNYI YE ${ }^{1,2,3}$, CHENXI ZHANG ${ }^{1,2,3}$, JIE ZHOU $^{1}$, JUN CHENG $^{2,3}$, ZHEN LV $^{1,2,3}$, \\ LIN ZHOU ${ }^{2,3}$, HAIYANG XIE ${ }^{2,3}$, JIAN WU ${ }^{1,2,3}$ and SHUSEN ZHENG ${ }^{1,2,3}$ \\ ${ }^{1}$ Division of Hepatobiliary and Pancreatic Surgery, Department of Surgery, First Affiliated Hospital, School of Medicine, \\ Zhejiang University; ${ }^{2}$ Key Laboratory of Combined Multi-Organ Transplantation, Ministry of Public Health; \\ ${ }^{3}$ Key Laboratory of Organ Transplantation, Hangzhou, Zhejiang 310003, P.R. China
}

Received October 3, 2013; Accepted February 18, 2014

DOI: $10.3892 / \mathrm{mmr} .2014 .2032$

\begin{abstract}
Ischemia reperfusion injury (IRI) occurs in almost every liver surgery and is associated with the reduction of the liver blood flow. Ischemia impairs liver function and can even cause liver failure following surgery. The present study aimed to identify a new molecular target allowing the reduction of IRI and explore the related cellular mechanism. Adenovirus $\left(\sim 2.5 \times 10^{12}\right.$ viral particles) bearing the human heat shock protein 27 (HSP27) gene was injected into rat liver through the ileocecal vein. Five days following the injection, ischemia was induced by clamping the median and left portal veins, hepatic arteries and bile ducts. The levels of alanine transaminase (ALT), aspartate aminotransferase (AST), glutathione (GSH) and superoxide dismutase (SOD) were measured. The infiltration of inflammatory cells and the expression of pro-inflammatory factors were investigated. The number of regulatory $\mathrm{T}$ cells (Tregs) was measured by flow cytometry. At $2 \mathrm{~h}$ following reperfusion, the group injected with HSP27 had the highest level of ALT and AST, followed by the group injected with HSP27 and treated with gadolinium trichloride $\left(\mathrm{GdCl}_{3}\right)$, the empty vector-injected and the vector+ $\mathrm{GdCl}_{3}$ groups. The HSP27 group also had the lowest levels of the oxidative stress-protective factors SOD and GSH, and the highest levels of pro-inflammatory factors. The number of Tregs was reduced in the groups injected with HSP27. In conclusion, the human HSP27 protein can effectively accelerate liver damage at the early stages of IRI in rats. Tregs might play a critical role in HSP27-induced liver injury.
\end{abstract}

Correspondence to: Professor Shusen Zheng or Professor Jian Wu, Division of Hepatobiliary and Pancreatic Surgery, Department of Surgery, First Affiliated Hospital, School of Medicine, Zhejiang University, 79 Qingchun Road, Hangzhou, Zhejiang 310003, P.R. China

E-mail: shusenzheng@zju.edu.cn

E-mail:drwujian@hotmail.com

Key words: heat shock protein 27, ischemic reperfusion injury, regulatory $\mathrm{T}$ cells, liver

\section{Introduction}

Ischemia reperfusion injury (IRI) is a common complication observed in patients who undergo liver resection or transplantation, or suffer from toxic liver injury and veno-occlusive diseases $(1,2)$. It is the major cause of the increasing mortality reported after liver injury, as it is linked with kidney, lung and other organs (3). Previous studies proposed that the excessive inflammatory response potentiated by the immune system is responsible for IRI $(4,5)$. Free radicals, interleukins and other inflammatory factors are released and cause damage to the local tissue. Therefore, inhibition of the inflammatory response may reduce IRI damage $(6,7)$. Numerous protective strategies against IRI have been adopted, including therapeutic hypothermia and ischemic preconditioning (8). However, few of them focus on the role of regulatory T cells (Tregs) during the process of liver IRI.

Tregs are a subpopulation of T cells that plays a critical role in the modulation of the immune system and of self-tolerance (9). Specifically, Tregs suppress immune responses mediated by other cell types, and in order to increase the immune response, they are downregulated during infection. It was shown that Tregs ameliorate kidney injury caused by IRI (10), an effect that may result from their immunosuppressive ability. Heat shock proteins (HSPs) are involved in numerous physiological processes, particularly stress. HSP27, a member of the HSP family, is able to mediate cytoskeletal stability and prevent cell apoptosis (11). Although in a recent study, the use of HSP27 proved to be helpful for tumor detection and diagnosis (12), our understanding of the roles of human HSP27 on reperfusion injury, particularly with regards to cell and tissue protection, is limited. A few studies on animal models showed that HSP27 confers protection from reperfusion injury $(13,14)$. Chen et al $(15)$ reported that mice overexpressing human HSP27 are protected from IRI. However, how the immune system affects IRI remains unclear. This study aimed to explore the interaction of HSP27 with Tregs and its effect on IRI.

\section{Materials and methods}

Animal procedures. Male Wistar rats (body weight, 200-230 g) were purchased from the Shanghai Animal Center (Chinese Academy of Science, Shanghai, China). The rats were kept in 
standard animal care conditions and bred with rat chow and water. These procedures were approved by the Animal Care Committee of the Zhejiang University, in accordance with the Principles of Laboratory Animal Care (NIH publication 85-23, revised 1985).

To clone the human HSP27 full length gene (NCBI Reference Sequence: NM_001540.3; open reading frame), HSP27 cDNA were amplified from a HCC cDNA library using the following primers: Forward, ACTGCTCGAGGCCACCATGACCGAGCGCCGCG and reverse, TACTGAATTCTTACTTGGCGGCAGTCTCAT). The PCR product was inserted into the XhoI/EcoRI site of the pIRES2-EGFP vector (Catalog \#6029-1; Clontech Laboratories, Inc., Mountain View, CA, USA) and sequenced. Subsequently, $H S P 27$ as well as the vector were packed with adenovirus vectorpAD/CMV/V5-DEST (Shanghai R\&S Biotechnology Co., Ltd., Shanghai, China). The human HSP27 gene was synthesized and inserted into the genome of the inactivated adenovirus. The adenovirus was amplified within human embryonic kidney 293 (HEK 293) cells and dissolved in phosphate-buffered saline. Rats received anesthetic ( $4 \%$ chloral hydrate, $0.7 \mathrm{ml} / 100 \mathrm{~g}$ ) intraperitoneally, and a midline laparotomy was performed. The ileocecal vein (a branch of the portal vein) was exposed, and $0.5 \mathrm{ml}$ of liquid containing $2.5 \times 10^{12}$ viral particles (VP) were injected into the rats via the ileocecal vein. The abdominal cavity was closed with 3-0 silk sutures. Five days later, the abdominal cavity was opened again and the median, left portal vein, hepatic artery and bile ducts were clamped in order to induce partial hepatic ischemia. The right vessels and bile ducts were not impeded, and intestinal congestion was thus avoided. A sixty-minute blockage was considered safe for the animals, since it did not cause severe liver failure or death. One hour later, the clip was removed to initiate hepatic reperfusion. The abdominal cavity was then closed with 3-0 silk sutures.

Animals were placed into 4 groups, all of which shared in common the induction of partial hepatic ischemia/ischemia reperfusion (IR) during the second surgery 5 days later: i) HSP27 group, where adenovirus bearing the HSP27 gene was injected; ii) HSP27+gadolinium trichloride $\left(\mathrm{GdCl}_{3}\right)$ group, where adenovirus bearing the HSP27 gene was injected along with $\mathrm{GdCl}_{3}$ (Sigma-Aldrich, St. Louis, MO, USA) through the ileocecal vein; iii) vector group, where adenovirus without the $H S P 27$ gene (empty vector) was injected; and iv) vector+ $\mathrm{GdCl}_{3}$ group, where $\mathrm{GdCl}_{3}$ was injected along with the empty vector.

All animals were sacrificed at different times after reperfusion $(0.5,2,4,12$ and $24 \mathrm{~h} ; \mathrm{n}=6$ in each group per time point). Blood samples were collected from the inferior vena cava of the rats for biochemical examination and flow cytometry. The liver of each rat was harvested, and one part was immediately frozen in liquid nitrogen and stored at $-80^{\circ} \mathrm{C}$ to be further analyzed by quantitative polymerase chain reaction (qPCR), and another part was fixed in formalin for hematoxylin-eosin staining.

Alanine transaminase (ALT), aspartate aminotransferase (AST), glutathione (GSH) and superoxide dismutase (SOD) assays. Blood samples were immediately centrifuged, and serum was collected and stored at $-80^{\circ} \mathrm{C}$ for the ALT and AST assays. A total of $100 \mu \mathrm{l}$ of each sample was diluted in $500 \mu \mathrm{l}$ double-distilled water. These dilutions were used to assess ALT and AST using an automated clinical analyzer (7600; Hitachi, Tokyo, Japan).
Liver damage was evaluated by measuring the GSH and SOD levels in the liver tissue homogenate using the corresponding commercial kits (Nanjing Jiancheng Bioengineering Institute, Nanjing, China) according to the manufacturer's instructions.

Hematoxylin-eosin staining. Explanted rat livers were fixed in $10 \%$ formalin for 5 days. After automated dehydration, the samples were embedded in paraffin, sectioned $(4 \mu \mathrm{m})$ and stained with hematoxylin-eosin. Sections were examined under a light microscope (BX41; Olympus Optical Co., GmbH, Hamburg, Germany).

Flow cytometry. Rat blood was harvested at various time points following reperfusion. Peripheral blood mononuclear cells were isolated using rat lymphocyte separation medium (Borunlaite Science and Technology Co., Ltd., Beijing, China). Following cell surface staining with fluorescein isothiocyanate (FITC)-conjugated anti-rat anti-CD4 and allophycocyanin (APC)-conjugated anti-rat anti-CD25 primary antibodies (eBioscience, San Diego, CA, USA), cells were fixed and permeabilized using Fix \& Perm reagents (Caltag Medsystems, Buckingham, UK) according to the manufacturer's instructions. Cells were then incubated with phycoerythrin (PE)-conjugated anti-mouse/rat anti-forkhead box protein P3 (Foxp3) as the secondary antibody (eBioscience). Stained cells were analyzed using a flow cytometer (FC500; Beckman Coulter, Inc., Brea, CA, USA) and the CellQuest software (BD Biosciences, Franklin Lakes, NJ, USA).

$q P C R$. We extracted total RNA from each frozen sample using the TRIzol ${ }^{\circledR}$ reagent (Invitrogen Life Technologies, Carlsbad, CA, USA) according to a standard protocol. Complementary DNA (cDNA) was synthesized from the extracted RNAs using Moloney murine leukemia virus reverse transcriptase (Promega Corp., Madison, WI, USA). The following qPCR primers were designed based on the reported DNA sequences: GAPDH (Gene ID: 24383) forward, 5'-GGGCTCTCTGCT CCTCCCTGTTCT and reverse, 5'-G CCGCCTGCTTC ACCACCTTC; macrophage inflammatory protein-2 (MIP-2) (Gene ID: 114105) forward, 5'-CCTCCAGCAAGC TCCC TCCTGT and reverse, 5'-GTGGGGTCCTGG AGGGGTCAC; monocyte chemotactic protein-1 (MCP-1) (Gene ID: 24770) forward, 5'-ACAGAGGCCAGCCCAGAAACCA and reverse, 5'-ACAGGCCCAGAAGCGTGACAGA. The PCR reaction was performed in a final volume of $10 \mu \mathrm{l}$, containing SYBR-Green PCR Master mix (containing SYBR ${ }^{\circledR}$ Green I dye, AmpliTaq Gold ${ }^{\circledR}$ DNA Polymerase, dNTPs with dUTP, Passive Reference 1 and optimized buffer; Applied Biosystems). The amplifications were conducted in an ABI 7500 Real-Time PCR system (Applied Biosystems, Foster City, CA, USA) with the following thermal cycling conditions: $95^{\circ} \mathrm{C}$ for $10 \mathrm{~min}$, followed by 40 cycles of $95^{\circ} \mathrm{C}$ for $15 \mathrm{sec}$ and $60^{\circ} \mathrm{C}$ for $1 \mathrm{~min}$. GADPH was used as an internal control. Samples were assayed in triplicate. Quantification of the mRNAs from the amplified products was performed using the comparative threshold cycle method as described in the manufacturer's manual.

\section{Results}

Inflammatory infiltration. All rats in this study survived ischemia and reperfusion. Fig. 1 shows hematoxylin-eosin stained 

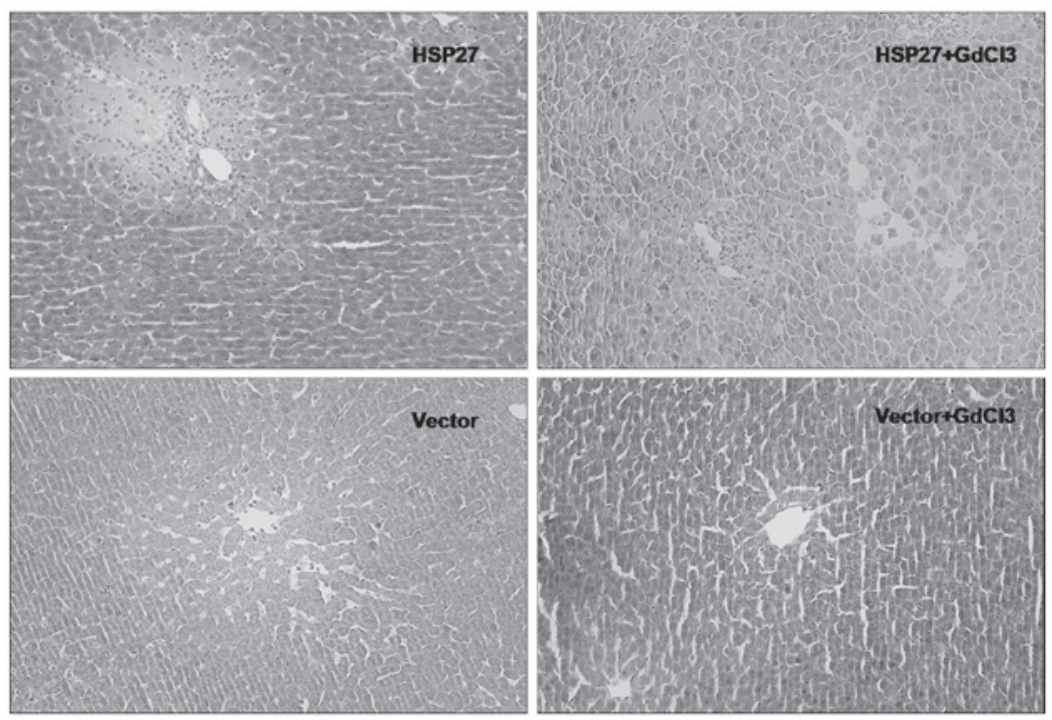

Figure 1. Inflammatory cells infiltrated the liver $2 \mathrm{~h}$ after ischemia and reperfusion. The HSP27 group showed the highest number of inflammatory cells. HSP27, heat shock protein $27 ; \mathrm{GdCl}_{3}$, gadolinium trichloride.

A

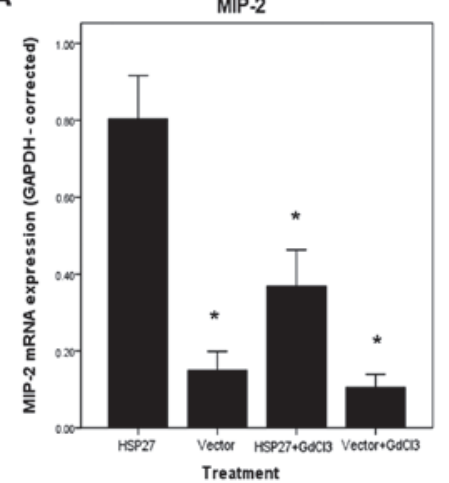

B

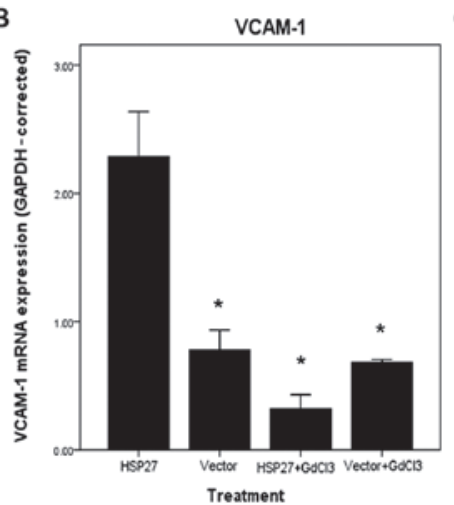

c

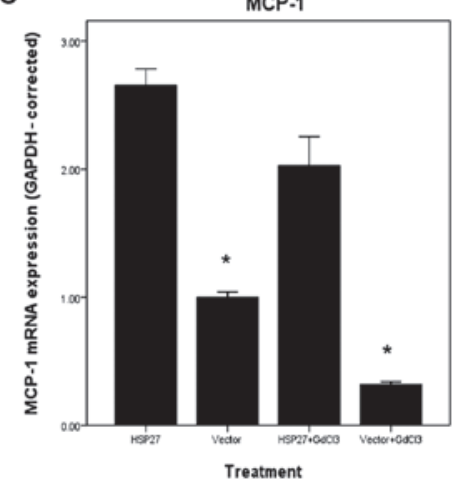

Figure 2. Expression of inflammation-related factors in the liver $2 \mathrm{~h}$ following reperfusion. (A) Macrophage inflammatory protein-2 (MIP-2), (B) vascular cell adhesion protein-1 (VCAM-1) and (C) monocyte chemotactic protein-1 (MCP-1) levels were significantly increased in the heat shock protein 27 (HSP27) group. ${ }^{*} \mathrm{P}<0.05$ vs. HSP27; ${ }^{\text {P }}<0.05$ vs. HSP27+gadolinium trichloride $\left(\mathrm{GdCl}_{3}\right)$.

liver sections following liver ischemia and reperfusion of the liver for $2 \mathrm{~h}$. Inflammatory cell infiltrations were more severe in the HSP27 group. In the control group, where rats were injected with an empty vector, reduced leukocyte infiltration was observed around the blood vessels. In the vector treated group, there were also fewer inflammatory cells compared to the HSP27 group. The vector $+\mathrm{GdCl}_{3}$ group was the least infiltrated with inflammatory cells.

Liver injury following IR. We found that the AST level was consistent with the inflammation levels in the different groups. The highest level was observed in the HSP27 group (data not shown). The combination of $\mathrm{HSP} 27$ and $\mathrm{GdCl}_{3}$ appeared to protect from IRI compared to the HSP27 group. The liver injury was more severe in the HSP27-injected groups (HSP27 and $\mathrm{HSP} 27+\mathrm{GdCl}_{3}$ ) compared to groups injected with the empty vector (vector and vector+ $\mathrm{GdCl}_{3}$ ). HSP27 appeared thus to exacerbate IRI at $2 \mathrm{~h}$ following reperfusion. The liver function was best in the vectors $+\mathrm{GdCl} 3$ treated group. Twenty four hours after ischemia, AST was still detected at a relatively high level in the vector group, while its level returned to normal in the other three groups. These results indicate that HSP27 might have a certain protective effect at later stages of ischemia.

Assessment of oxidative stress. The antioxidant enzyme SOD, as well as GSH, play an important role in protecting cells from oxidative stress and attenuating the effects of injury. They are crucial for maintaining the balance between reactive oxygen species and antioxidants, which also help prevent damages caused by oxidative stress (16). The depletion of SOD and GSH occurs at the initial phase of liver necrosis (17). The levels of the stress-related markers SOD and GSH were significantly decreased in the HPS27 group compared to the vector $+\mathrm{GdCl}_{3}$ group, indicating that $\mathrm{HSP} 27$ may exacerbate reperfusion injury in rats. Overall, the SOD and GSH levels were consistent with the AST level, i.e. the more damaged the liver was, the lower were the SOD and GSH levels.

Amplification of inflammatory factor genes. Fig. 2 shows the changes in expression of pro-inflammatory factors in the four groups of rats following reperfusion. The mRNA levels of genes encoding the vascular cell adhesion protein-1 (VCAM-1), 

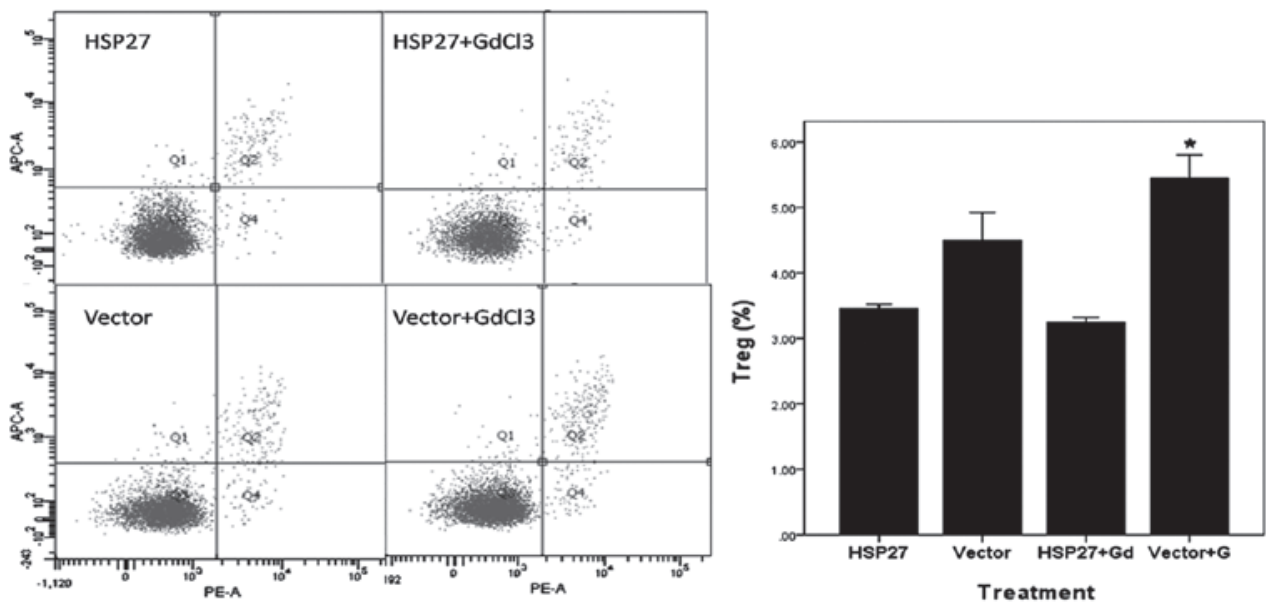

Figure 3. The percentage of regulatory T cells (Tregs) in the peripheral blood of rats from different groups. The number of Tregs was decreased in the groups injected with the heat shock protein 27 (HSP27) gene. ${ }^{*} \mathrm{P}<0.05$ vs. the $\mathrm{HSP} 27$ group. $\mathrm{GdCl}_{3}$, gadolinium trichloride.

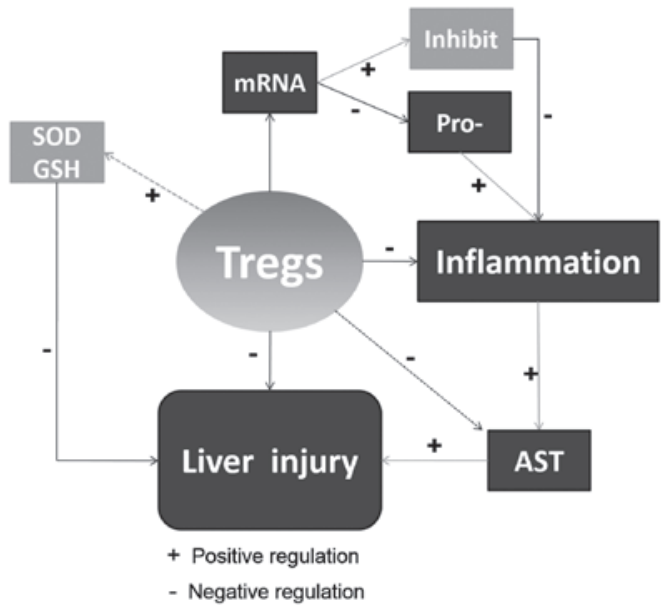

Figure 4. Regulatory T cells (Tregs) play a central role during the period following ischemia and reperfusion. In our study, Tregs were associated with decreased superoxide dismutase (SOD) and glutathione (GSH) levels, increased levels of pro-inflammatory factors and aspartate aminotransferase (AST), as well as with enhanced liver injury.

MCP-1 and MIP-2 were increased in rats of the HSP27 group compared with the other groups. These results suggested that HSP27 enhances the inflammatory response in the rat liver following IR.

Treg levels in the serum. Fig. 3 shows the levels of Tregs in the different groups after $2 \mathrm{~h}$ of IR. Tregs were fewer in number in the HSP27 groups, which might be the cause of the more severe inflammation in these groups. The percentage of Tregs in the serum of rats from the HSP27 group was much lower compared to that observed in the vector and vector $+\mathrm{GdCl}_{3}$ groups. Therefore, HSP27 may suppress Tregs. By contrast, in the groups that were not injected with $H S P 27$, the percentage of Tregs was higher compared to the groups injected with HSP27.

\section{Discussion}

The major finding of our study was that rats overexpressing the HSP27 gene can exacerbate liver injury following IR. This might be partly due to the downregulation of Tregs (Fig. 4). In addition to the regular function of HSP in folding and unfolding proteins, induction of its expression in response to stress has been suggested to be protective for the cells $(18,19)$. A proteomic analysis showed that the blockage of chaperones such as HSP may contribute to increased rates of apoptosis and necrosis (20). Hence, we investigated the roles of HSP in response to hepatocyte injury.

In this study, we transfected rat hepatocytes with an adenovirus bearing the human HSP27 gene, so that its expression would increase when IRI occurs. We chose to use the human instead of the rat HSP27 gene for two reasons: first, Chen et al (15) showed that overexpressed HSP27 in mice can protect liver from IRI, and we thus aimed to investigate whether the human gene may have a certain protective function in rats as well. Second, in case this proved to be true, we may assume that the human HSP27 protein has a universal protective function in liver IRI, which is very promising for clinical patients who undergo liver surgery.

Although the detailed mechanism leading to IRI has not been fully explored yet, it is widely accepted that several factors contribute to IRI, including free radicals, cytokines and numerous pro-inflammatory factors (21). From this perspective, liver injury following ischemia is initiated by inflammatory infiltration and correlates with the severity of inflammation, and the degree of liver function can be determined by the inflammatory response following reperfusion (4). In the present study, the increase of inflammatory infiltration was corroborated by hematoxylin-eosin staining. We found that inflammation was more severe in the HSP27 group, followed by that observed in the $\mathrm{HSP} 27+\mathrm{GdCl}_{3}$, vector and vector+ $\mathrm{GdCl}_{3}$ groups.

We further investigated liver function following IRI in the different groups. A lower AST level was observed in both groups treated with the empty vector. As inflammatory cells were recruited, the increase in necrosis, apoptosis and other processes of cell death resulted in severe damage of liver cells. Therefore, we conclude that the human HSP27 protein induces inflammation in the rat liver at the early stage of IRI. Nevertheless, the observation that AST remained at a relatively high level in the empty vector group at later stages of IRI, while it was decreased in the other three groups after $24 \mathrm{~h}$, is surprising. This might be due to the fact that the human HSP27 protein contributes to 
tissue repair at later stages of IRI. HSP27 apart from its role in attacking inflammatory cells at the early stage, might contribute in maintaining the liver structure at later stages; inflammation represents indeed a defense mechanism, protecting the cells from further damage.

Oxygen, free radicals, cytokines and other pro-inflammatory factors are involved in the processes of liver ischemia and reperfusion (22). In the present study, SOD and GSH levels in rat liver tissues of the HSP27 group were lower compared to the vector $+\mathrm{GdCl}_{3}$ group, indicating that $\mathrm{HSP} 27$ has an unfavorable antioxidant activity.

Treatment with HPS27 has been associated with decreased expression of transforming growth factor- $\beta$ (TGF- $\beta$ ). The cytokine TGF- $\beta$ is important for the assembly of liver cells and promotes growth of hepatocytes $(23,24)$. In our study, overexpression of the human $H S P 27$ gene in rat liver was associated with a decrease in the level of the TGF- $\beta$ mRNA, suggesting that HSP27 has a negative role in liver protection at the early stage of IRI. The increased mRNA expression of pro-inflammatory factors such as VCAM-1, MCP-1 and MIP-2 further revealed that reperfusion injury is associated with the upregulation of signaling pathways involved in inflammatory responses. It was previously demonstrated that $\mathrm{T}$ cells are key mediators of inflammatory responses in the liver following IR (25), and that depletion of $\mathrm{T}$ cells confers protection from IRI (26). Compared to other subgroups of T cells, Tregs can directly suppress the activation of monocytes/macrophages, and can also produce inhibitory cytokines (27). The deprivation or downregulation of Tregs may lead to inflammatory response and cause liver damage. In our study, we demonstrated that Tregs are reduced in the HSP27 group, which led to reduced protection of the liver from IRI.

In conclusion, numerous factors are related to liver IRI. For example, hepatic stellate cells may also play a crucial role in the progression of IRI (28). The human HSP27 gene does not protect rat liver from IRI at the early stages, however, it might exert protective effects at a later stage.

\section{Acknowledgements}

This study was supported by grants from the Zhejiang Provincial Natural Science Foundation for Young Distinguished Scholars (no. R2110125), the National Natural Science Foundation of China (no. 81272281) and the National High Technology Research and Development Program 863 of China (no. 2012AA021002). The funding institutes had no role in the study design, collection, analysis and interpretation of data, preparation or submission of the manuscript.

\section{References}

1. Teoh NC and Farrell GC: Hepatic ischemia reperfusion injury: pathogenic mechanisms and basis for hepatoprotection. J Gastroenterol Hepatol 18: 891-902, 2003.

2. Fondevila C, Busuttil RW and Kupiec-Weglinski JW: Hepatic ischemia/reperfusion injury - a fresh look. Exp Mol Pathol 74: 86-93, 2003.

3. Wobbes T, Bemelmans BL, Kuypers JH, Beerthuizen GI and Theeuwes AG: Risk of postoperative septic complications after abdominal surgical treatment in relation to perioperative blood transfusion. Surg Gynecol Obstet 171: 59-62, 1990.
4. Daemen MA, de Vries B and Buurman WA: Apoptosis and inflammation in renal reperfusion injury. Transplantation 73: 1693-1700, 2002.

5. Serracino-Inglott F, Habib NA and Mathie RT: Hepatic ischemia-reperfusion injury. Am J Surg 181: 160-166, 2001.

6. Pundik S, Xu K and Sundararajan S: Reperfusion brain injury: focus on cellular bioenergetics. Neurology 79: S44-S51, 2012.

7. Song X, Zhang N, Xu H, Cao L and Zhang H: Combined preconditioning and postconditioning provides synergistic protection against liver ischemic reperfusion injury. Int J Biol Sci 8: 707-718, 2012.

8. Gurusamy KS, Gonzalez HD and Davidson BR: Current protective strategies in liver surgery. World J Gastroenterol 16: 6098-6103, 2010.

9. Hori S, Nomura T and Sakaguchi S: Control of regulatory T cell development by the transcription factor Foxp3. Science 299: 1057-1061, 2003.

10. Lai LW, Yong KC and Lien YH: Pharmacologic recruitment of regulatory $\mathrm{T}$ cells as a therapy for ischemic acute kidney injury. Kidney Int 81: 983-992, 2012.

11. Rane MJ, Pan Y, Singh S, et al: Heat shock protein 27 controls apoptosis by regulating Akt activation. J Biol Chem 278: 27828-27835, 2003.

12. Nagata Y, Kudo M, Nagai T, et al: Heat shock protein 27 expression is inversely correlated with atrophic gastritis and intraepithelial neoplasia. Dig Dis Sci 58: 381-388, 2013.

13. Park SW, Chen SW, Kim M, D'Agati VD and Lee HT: Human heat shock protein 27 -overexpressing mice are protected against acute kidney injury after hepatic ischemia and reperfusion. Am J Physiol Renal Physiol 297: F885-F894, 2009.

14. Kim M, Park SW, Kim M, Chen SW, Gerthoffer WT, D'Agati VD and Lee HT: Selective renal overexpression of human heat shock protein 27 reduces renal ischemia-reperfusion injury in mice. Am J Physiol Renal Physiol 299: F347-F358, 2010.

15. Chen SW, Park SW, Kim M, Brown KM, D'Agati VD and Lee HT: Human heat shock protein 27 overexpressing mice are protected against hepatic ischemia and reperfusion injury. Transplantation 87: 1478-1487, 2009.

16. Taniguchi M, Takeuchi T, Nakatsuka R, Watanabe T and Sato K: Molecular process in acute liver injury and regeneration induced by carbon tetrachloride. Life Sci 75: 1539-1549, 2004.

17. Williams AT and Burk RF: Carbon tetrachloride hepatotoxicity: an example of free radical-mediated injury. Semin Liver Dis 10: 279-284, 1990.

18. Sharma A, Upadhyay AK and Bhat MK: Inhibition of Hsp27 and Hsp40 potentiates 5-fluorouracil and carboplatin mediated cell killing in hepatoma cells. Cancer Biol Ther 8: 2106-2113, 2009.

19. O'Neill S, Ross JA, Wigmore SJ and Harrison EM: The role of heat shock protein 90 in modulating ischemia-reperfusion injury in the kidney. Expert Opin Investig Drugs 21: 1535-1548, 2012.

20. Tiriveedhi V, Conzen KD, Liaw-Conlin J, et al: The role of molecular chaperonins in warm ischemia and reperfusion injury in the steatotic liver: a proteomic study. BMC Biochem 13: 17, 2012.

21. Montalvo-Jave EE, Escalante-Tattersfield T, Ortega-Salgado JA, Pina E and Geller DA: Factors in the pathophysiology of the liver ischemia-reperfusion injury. J Surg Res 147: 153-159, 2008.

22. Weerachayaphorn J, Chuncharunee A, Jariyawat S, et al: Protection of centrilobular necrosis by Curcuma comosa Roxb. in carbon tetrachloride-induced mice liver injury. J Ethnopharmacol 129: 254-260, 2010.

23. Breitkopf K, Godoy P, Ciuclan L, Singer MV and Dooley S: TGF-beta/Smad signaling in the injured liver. Z Gastroenterol 44: 57-66, 2006.

24. Michalopoulos GK: Liver regeneration. J Cell Physiol 213: 286-300, 2007.

25. Zwacka RM, Zhang Y, Halldorson J, Schlossberg H, Dudus L and Engelhardt JF: CD4(+) T-lymphocytes mediate ischemia/reperfusion-induced inflammatory responses in mouse liver. J Clin Invest 100: 279-289, 1997.

26. Yokota N, Daniels F, Crosson J and Rabb H: Protective effect of $\mathrm{T}$ cell depletion in murine renal ischemia-reperfusion injury. Transplantation 74: 759-763, 2002.

27. Taams LS, van Amelsfort JM, Tiemessen MM, et al: Modulation of monocyte/macrophage function by human CD4+CD25+ regulatory T cells. Hum Immunol 66: 222-230, 2005.

28. Jameel NM, Thirunavukkarasu C, Murase N, et al: Constitutive release of powerful antioxidant-scavenging activity by hepatic stellate cells: protection of hepatocytes from ischemia/reperfusion injury. Liver Transpl 16: 1400-1409, 2010. 